\title{
Sensitivity Study of Manoeuvring Motion of Ships using Whole Ship Model
}

\author{
Fakhri Akbar Ayub ${ }^{1, a, *}$ Yoshitaka Furukawa ${ }^{2, b}$, and Hiroshi Ibaragi 2,c \\ ${ }^{1}$ Department of Maritime Engineering, Graduate School of Engineering, Kyushu University, \\ Fukuoka, Japan \\ ${ }^{2}$ Department of Marine Systems Engineering, Faculty of Engineering, Kyushu University, Fukuoka, \\ Japan \\ a.2TE16356Y@kyushu-u.ac.jp,b.furukawa@nams.kyushu-u.ac.jp,c.ibaragi@nams.kyushu- \\ u.ac.jp \\ *corresponding author
}

Keywords: Sensitivity Analysis; Monte Carlo Simulation; Ship Manoeuvrability; Hydrodynamic Coefficients

Abstract: Accurate estimation of a ship manoeuvrability is important issue as for the safety of ships at the sea. To accomplish the prediction of ship manoeuvrability with high accuracy using mathematical models for hydrodynamic forces acting on a ship, the values of hydrodynamic coefficients included in the mathematical model should have accurate values. Generally, ship manoeuvrability is evaluated by standard manoeuvring tests such as turning ability, course-keeping and yaw-checking abilities and so on. By investigating the sensitivity of each hydrodynamic coefficient on the results of the standard manoeuvring tests, it is possible to understand which coefficients should be mostly paid attention when they are obtained by numerical simulations. In this paper, sensitivity analysis of all the hydrodynamic derivatives using whole ship model is performed by using the conventional Monte Carlo Simulation (MCS) to evaluate the influence of coefficient on performance indices such as advance, transfer, tactical diameter and 1st and 2 nd overshoot angles. The values of hydrodynamic derivatives are varied with the range of standard deviation which is assumed as $10 \%$ of their mean value. The results of simulation showed that which hydrodynamic coefficients have dominant effect on the performance indices of standard manoeuvring tests.

\section{INTRODUCTION}

Accurate estimation of ship manoeuvrability is important issue as for the safety of ships at the sea. Since criteria for manoeuvring characteristics were defined in the Standards for Ship Manoeuvrability as Res. 137(76) which was adopted by International Maritime Organization (IMO) [1]. In order to eliminate ships which have poor manoeuvrability, ship manoeuvring performance should be evaluated properly at design phase, not to build ships which cannot satisfy the criteria. Though there are several methods to evaluate ship manoeuverbility at the design stage, numerical simulation based 
on the mathematical models of hydrodynamic forces acting on a ship is one of the useful methods. In order to obtain prediction results with high accuracy, it is essential to use accurate values for hydrodynamic coefficients included in the mathematical models.

In addition, the manoeuvring standards require to prove that a newly-built ship can satisfy the criteria which are defined in fully loaded condition by conducting manoeuvring tests in calm environmental condition. However, sea trials are often conducted at ballast condition, because it is difficult to make fully loaded condition especially for dry cargo ships such as container carriers or bulk carriers. Furthermore, the influence of external disturbances should be excluded from measured results, unless the sea trial was conducted in completely calm conditions without current, wind and waves. Therefore, ship builders should estimate manoeuvring performance of the ship in fully loaded and calm environmental conditions considering the measured results. In this phase, the numerical simulation method takes an important role.

As mentioned above, the accuracy of hydrodynamic coefficients in mathematical models to represent hydrodynamic forces acting on a ship has crucial relevance with predicted manoeuvring characteristics. In general, these hydrodynamic coefficients are determined by conducting captive model tests. If it is not possible to carry out the captive model tests, the other methods to define the coefficients such as Computational Fluid Dynamics (CFD) calculations, database which contains the data of similar ship type or empirical formulae based on principal dimensions of a ship are often used. Even if captive model tests are conducted, the influence of errors in measurement or analysis on the hydrodynamic coefficients is not avoidable. Needless to say, CFD calculations, database or empirical formulae cannot provide true values for the coefficients. Accordingly, it is of importance to grasp the effects of the variation of these coefficients on predicted manoeuvring characteristics.

From the viewpoints, the Manoeuvring Committee in 28th International Towing Tank Convention carried out three kinds of sensitivity analyses for ship manoeuvrability [2]. One of the authors conducted the first application of the sensitivity analysis as the member of the committee using whole ship model for the KLVCC2 [3][4]. Second application was performed based on the regression analysis of a linear manoeuvring model test [5]. The third application was carried out based on the 4DOF mathematical model of a container ship S175 presented by Son and Nomoto [6]. These sensitivity analyses presented by the 28th ITTC Manoeuvring Committee have its own advantages and disadvantages, nevertheless they have a same objective that is to show which coefficients have a dominant effect on ship manoeuvrability. Wang et al. [8] proposed to perform spiral test instead of normal turning test to conduct sensitivity analysis. They indicated that non-relevant hydrodynamic coefficient could be deleted by doing sensitivity analysis. According to their comparison between the simulation results of the original and simplified mathematical model for turning test and zigzag test, mathematical model can be simplified significantly based on the sensitivity analysis.

Conventional Monte Carlo simulation is useful to identify the sensitivity for manoeuvring performance of each hydrodynamic coefficient. By doing the sensitivity analysis, we can determine which coefficients have significant effect on predicted results or not. It means that we can understand which coefficients should be mostly paid attention when we perform numerical simulations. Woodward [7] investigated the source of uncertainty on the inter-facility test. In his research, Monte Carlo simulation is presented to evaluate the propagation of uncertainty. The results of his study on KLVCC1 show that hydrodynamic coefficients in normal probability distribution generate overshoot angles of zigzag test in a Weibull distribution. Dash et al (2015) proposed a conventional Monte Carlo simulation to analyse the uncertainties in MMG type of $8 \mathrm{DoF}$ manoeuvring mathematical model for DTMB 5415 ship. Hermite polynomial chaos (HPC) expansions are used to fit the probability densities of the mathematical model coefficient. Monte Carlo Simulation method presented to propagate the uncertainty for full-scale zigzag manoeuvre. It was shown that the mathematical model uncertainty is higher than the experimental uncertainty [9]. 
The aim of this paper is to investigate the sensitivity of all the hydrodynamic derivatives used in a whole ship model on predicted manoeuvring performance based on the conventional Monte Carlo Simulation (MCS). Turning ability and course-keeping and yaw-checking abilities are mainly focused on. The variation of performance indices such as advance, transfer, tactical diameter, 1st and 2nd overshoot angles due to the change of the value of hydrodynamic coefficients are evaluated to show the influence of the coefficients on them. Simulation results showed that which hydrodynamic coefficients have dominant effect on the performance indices of standard manoeuvring tests.

\section{MATHEMATICAL MODEL}

To conduct sensitivity analysis, whole ship model for the KVLCC2 presented in [3][4] is used as a mathematical model for ship manoeuvring motion. The principal particulars of the KVLCC2 are shown in Table I.

The manoeuvring motions of a surface ship such as surge, sway and yaw can be described by the following equation,

$$
\left.\begin{array}{rl}
\left(m^{\prime}-X_{\dot{u}}^{\prime}\right) \dot{u}^{\prime} & =f_{1}\left(\eta, v^{\prime}, r^{\prime}, \delta\right), \\
\left(m^{\prime}-Y_{\dot{v}}^{\prime}\right) \dot{v}^{\prime}+\left(m^{\prime} x_{G}^{\prime}-Y_{\dot{r}}^{\prime}\right) \dot{r}^{\prime} & =f_{2}\left(\eta, v^{\prime}, r^{\prime}, \delta\right), \\
\left(m^{\prime} x_{G}^{\prime}-N_{\dot{v}}^{\prime}\right) \dot{v}^{\prime}+\left(I_{z}^{\prime}-N_{\dot{r}}^{\prime}\right) \dot{r}^{\prime} & =f_{3}\left(\eta, v^{\prime}, r^{\prime}, \delta\right)
\end{array}\right\}
$$

Table 1: Principal Particulars of KVLCC2

\begin{tabular}{|c|c|}
\hline$L$ & $320 \mathrm{~m}$ \\
\hline$B$ & $58 \mathrm{~m}$ \\
\hline$D$ & $20.8 \mathrm{~m}$ \\
\hline$C_{b}$ & 0.8098 \\
\hline Scale ratio & 1.0 \\
\hline
\end{tabular}

where, $\mathrm{m}$ is the mass of the ship, $\mathrm{I} \_\mathrm{z}$ is the moment of inertia about the vertical axis which through ship's centre of gravity (COG); $x_{-} G$ is the longitudinal coordinate of COG; $u, v$ and $r$ denote the surge speed, sway speed and yaw rate respectively; $\mathrm{X}_{-} \mathrm{u}^{\cdot}, \mathrm{Y}_{-} \mathrm{v}^{\cdot}, \mathrm{N}_{-} \mathrm{r}^{\cdot}$ etc., are hydrodynamic derivatives for acceleration components. Functions $f_{-} 1, f_{-} 2$ and $f \_3$ shown in the right-hand side of (1) are expressed as follows.

$$
\begin{aligned}
f_{1}\left(\eta, v^{\prime}, r^{\prime}, \delta\right) & =X_{\eta}^{\prime}(1-\eta)+X_{\eta \eta}^{\prime}(1-\eta)^{2} \\
& +X_{\eta \eta \eta}^{\prime}(1-\eta)^{3}+X_{v v}^{\prime} v^{\prime 2} \\
& +\left(X_{r r}^{\prime}+m^{\prime} x_{G}^{\prime}\right) r^{\prime 2}+X_{\delta \delta}^{\prime} \delta^{2} \\
& +\left(X_{v r}^{\prime}+m^{\prime}\right) v^{\prime} r^{\prime}+X_{v v \eta}^{\prime} v^{\prime 2}(1-\eta) \\
& +X_{\delta \delta \eta}^{\prime} \delta^{2}(1-\eta), \\
f_{2}\left(\eta, v^{\prime}, r^{\prime}, \delta\right) & =Y_{0}^{\prime}+Y_{v}^{\prime} v^{\prime}+Y_{v r r}^{\prime} v^{\prime} r^{\prime 2}+\left(Y_{r}^{\prime}-m^{\prime}\right) r^{\prime} \\
& +Y_{r r r}^{\prime} r^{\prime 2}+Y_{v v r}^{\prime} v^{\prime 2} r^{\prime}+Y_{\delta}^{\prime} \delta+Y_{\delta \delta \delta}^{\prime} \delta^{3} \\
& +Y_{\eta}^{\prime}(1-\eta)+Y_{\eta \eta}^{\prime}(1-\eta)^{2} \\
& +Y_{\delta \eta}^{\prime} \delta(1-\eta)+Y_{\delta \eta \eta}^{\prime} \delta(1-\eta)^{2}, \\
f_{3}\left(\eta, v^{\prime}, r^{\prime}, \delta\right) & =N_{0}^{\prime}+N_{v}^{\prime}+N_{v r r}^{\prime} v^{\prime} r^{\prime 2} \\
& +\left(N_{r}^{\prime}-m^{\prime} x_{G}^{\prime}\right) r^{\prime}+N_{r r r}^{\prime} r^{\prime 2} \\
& +N_{v v r}^{\prime} v^{\prime 2} r^{\prime}+N_{\delta}^{\prime} \delta+N_{\delta \delta \delta}^{\prime} \delta^{3} \\
& +N_{\eta}^{\prime}(1-\eta)+N_{\eta \eta}^{\prime}(1-\eta)^{2} \\
& +N_{\delta \eta}^{\prime} \delta(1-\eta)+N_{\delta \eta \eta}^{\prime} \delta(1-\eta)^{2},
\end{aligned}
$$


where, $\eta$ and $\delta$ indicate speed drop and rudder angle respectively. The superscript " ' " in the (1) and (2) refers to the non-dimensionalized parameters as follows;

$$
\left.\begin{array}{l}
m^{\prime}=\frac{m}{(1 / 2) \rho L^{3}}, \quad I_{z}^{\prime}=\frac{I_{z}}{(1 / 2) \rho L^{5}}, \quad x_{G}^{\prime}=\frac{x_{G}}{L}, \\
u^{\prime}, v^{\prime}=\frac{u, v}{U}, \quad r^{\prime}=\frac{r L}{U}, \\
\dot{u}^{\prime}, \dot{v}^{\prime}=\frac{L}{U^{2}}(\dot{u}, \dot{v}), \quad \dot{r}^{\prime}=\frac{L^{2}}{U^{2}} \dot{r}, \quad \eta=\frac{U}{U_{0}},
\end{array}\right\}
$$

where, $\rho$ and $L$ are the density of the water and ship length. $U$ and $U_{-} 0$ indicate ship speed and initial ship speed. The values of the hydrodynamic coefficients [3][4] used in (1) and (2) are shown Table II.

\section{SENSITIVITY ANALYSIS}

Sensitivity analysis can be described as how sensitively simulated results respond to the variation of parameters used in a mathematical model. As the first step, sensitivity for manoeuvring characteristics of each hydrodynamic coefficient was investigated. The value of each coefficient is changed up to $\pm 10 \%$ of the value presented in Table 2 while the other coefficients remained constant. The evaluated performance indices are,

Table 2: Manoeuvring Coefficients for whole ship model

\begin{tabular}{|l|r|l|l|l|r|}
\hline \multicolumn{2}{|c|}{ Surge Coeff. $\times \mathbf{1 0}^{\mathbf{5}}$} & \multicolumn{2}{c|}{ Sway Coeff. $\times \mathbf{1 0}^{5}$} & \multicolumn{2}{c|}{ Yaw Coeff. $\times \mathbf{1 0}^{5}$} \\
\hline$m-X_{u}$ & 2139.8 & $m-Y_{\dot{v}}$ & 3413.3 & $m x_{G}-N_{\dot{v}}$ & 2.8 \\
& & $m x_{G}-Y_{\dot{r}}$ & 138.7 & $I_{z}-N_{\dot{r}}$ & 210.9 \\
\hline$X_{\delta \delta}$ & -169.8 & $Y_{0}$ & -0.7 & $N_{0}$ & 4.9 \\
& & $Y_{\delta \delta}$ & 315.4 & $N_{\delta}$ & -186.2 \\
& & $Y_{\delta \delta}$ & 128.7 & $N_{\delta \delta}$ & -87.4 \\
\hline$X_{\eta}$ & -211.7 & $Y_{\eta}$ & 2.1 & $N_{\eta}$ & -10.2 \\
$X_{\eta \eta}$ & 223.9 & $Y_{\eta \eta}$ & -8.1 & $N_{\eta \eta}$ & -1.27 \\
$X_{\eta \eta \eta}$ & 16.8 & $Y_{\eta \eta \eta}$ & -396.2 & $N_{\eta \eta \eta}$ & 200.1 \\
$X_{\delta \delta \eta}$ & 157.5 & $Y_{\delta \delta \eta}$ & 95.6 & $N_{\delta \delta \eta}$ & -58.4 \\
\hline$X_{v v}$ & 354.1 & $Y_{v}$ & -2364.4 & $N_{v}$ & -732.9 \\
& & $Y_{v v v}$ & -3927.2 & $N_{v v v}$ & 356.4 \\
\hline$X_{r r}$ & -4.56 & $Y_{r}-m$ & -1292.1 & $N_{r}$ & -370.7 \\
& & $Y_{r r r}$ & -221.4 & $N_{r r r}$ & 11.7 \\
\hline$X_{v r}+\left(m+m_{y}\right)$ & 3543.1 & $Y_{v r r}$ & -148.8 & $N_{v r r}$ & 1014.0 \\
& & $Y_{v v r}$ & 2815.7 & $N_{v v r}$ & -1824.7 \\
\hline
\end{tabular}

Advance, transfer, tactical diameter, steady values of drift angle $\beta$, non-dimensional yaw rate $\mathrm{r}^{\prime}$ and speed drop U/U_ 0 in $35^{\circ}$ turning,

- 1 st and 2 nd overshoot angles in $10^{\circ} / 10^{\circ}$ zigzag manoeuvre,

- 1 st and 2 nd overshoot angles in $20^{\circ} / 20^{\circ}$ zigzag manoeuvre.

In this way dominant coefficients can be detected for each characteristic of a manoeuvre.

In the second step, Monte Carlo Simulation were carried out. The standard deviation of each hydrodynamic coefficient was assumed as $5 \%$ of the value of each hydrodynamic coefficient shown 
in Table II. The value of each coefficient was varied by the following equation in the Monte Carlo Simulation,

$$
D=\mu+\sigma \cdot n_{r}
$$

where, $\mathrm{D}$ gives varied value of each hydrodynamic coefficient. $\sigma$ and $\mu$ are the standard deviation and the original value of each hydrodynamic coefficient. $n \_r$ is a random number following normal distribution.

Using the combination of hydrodynamic coefficients generated by applying (4), one million times calculations were executed during the Monte Carlo Simulation process. The evaluated performance indices were same as those of the first step. Calculation results of the performance indices obtained by the Monte Carlo Simulation were processed by using " $\mathrm{R}$ " which is a free software environment for statistical computing.

\section{SENSITIVITY FOR MANOEUVRING CHARACTERISTIC OF EACH HYDRODYNAMIC DERIVATIVE}

In this section, the results of the first step of the sensitivity analysis are presented. Figure 1 shows the changes of the values of performance indices for turning ability with the variation of hydrodynamic coefficients. The changes of transfer due to the variation of $X^{\prime}, Y^{\prime}$ and $N^{\prime}$ derivatives are displayed in Figure 1(d)-(f). The value of transfer is not affected so much by the variation of $X^{\prime}$ and $Y^{\prime}$ derivatives. On the other hand, some of $N^{\prime}$ derivatives have significant influence on transfer comparing with $\mathrm{X}^{\prime}$ and $\mathrm{Y}^{\prime}$ derivatives. With the $\pm 10 \%$ variation of $\mathrm{N} \_\mathrm{r}^{\wedge^{\prime}}-\mathrm{m}^{\wedge^{\prime}} \mathrm{x} \mathrm{G}^{{ }^{\wedge}}, \mathrm{N} \delta^{{ }^{\prime \prime}}$ and $\mathrm{N} \_\mathrm{v}^{\wedge \prime}$, predicted values of transfer spread through the range of approximately $\pm 3 \%$.

Figure 1(a)-(c) and Figure 1(g)-(i) display the changes of advance and tactical diameter due to the variation of $\mathrm{X}^{\prime}, \mathrm{Y}^{\prime}$ and $\mathrm{N}^{\prime}$ derivatives. It is observed that the values of advance and tactical diameter are changed by the variation of $\mathrm{X}_{-} \mathrm{vr}^{\wedge}-\mathrm{m}^{\prime}$, but they are not so remarkable. As for $\mathrm{Y}^{\prime}$ derivatives, though the variation of advance is observed in Figure 1(b), their influence is not so much either. Notable changes of advance and tactical diameter appear for some of $\mathrm{N}^{\prime}$ derivatives. It can be said from these observations that $N_{-} \mathrm{r}^{\wedge^{\prime}}-\mathrm{m}^{\wedge^{\prime}} \mathrm{x} \mathrm{G}^{\wedge^{\prime}}$ and $\mathrm{N}_{-} \mathrm{v}^{{ }^{\prime \prime}}$ have a dominant effect on the advance, transfer and tactical diameter of a turning circle.

The changes of performance indices for course-keeping and yaw-checking abilities with the variation of hydrodynamic coefficients are shown in Figure 2. It is easily understood that overshoot angles of $10^{\circ} / 10^{\circ}$ zigzag and $20^{\circ} / 20^{\circ}$ zigzag are hardly affected by the variation of $\mathrm{X}^{\prime}$ derivatives. As same as the performance indices for turning ability, $N_{-} \mathrm{r}^{\wedge^{\prime}}-\mathrm{m}^{\wedge^{\prime}} \mathrm{x} \mathrm{G}^{{ }^{\prime}}$ and $\mathrm{N}_{-} \mathrm{v}^{\wedge^{\prime}}$ have biggest impact on the values of overshoot angles in zigzag manoeuvre. The values of them may exist within the range of approximately $\pm 20 \%$. Following the $\mathrm{N}^{\prime}$ derivatives, $\mathrm{Y}_{-} \mathrm{r}^{\wedge^{\prime}}-\mathrm{m}^{\wedge^{\prime}}$ and $\mathrm{Y}_{-} \mathrm{v}^{\wedge^{\prime}}$ also affect the overshoot angles.

According to the results of the first step of the sensitivity analysis, dominants coefficients for turning ability and course-keeping and yaw-checking abilities were picked up. However, strictly speaking, if the value of a certain hydrodynamic coefficient changes, the values of other coefficients which are closely related to the coefficient also fluctuate. Therefore, coupled variation of closely related hydrodynamic coefficients should be considered for strict investigation.

\section{SENSITIVITY ANALYSIS BASED ON MONTE CARLO SIMULATION}

The results of Monte Carlo Simulation conducted as the second step of the sensitivity analysis are presented in this section. The frequency distributions of the values of the performance indices for 
turning ability obtained from the Monte Carlo Simulation are shown Figure 3. The range of the values of advance, transfer and tactical diameter around the mean values of them are indicated in lateral axis and vertical axis represents their frequency. It seems that normal distribution can be assumed for the variation of advance, transfer and tactical diameter, though there are differences among the standard deviations of them. The standard deviation of transfer is relatively smaller than those of advance and tactical diameter.

Figure 4 shows the frequency distributions of 1 st and 2 nd overshoot angles for $10^{\circ} / 10^{\circ}$ zigzag manoeuvre and 1st overshoot angle for $20^{\circ} / 20^{\circ}$ zigzag manoeuvre. It is observed from the figure that the shape of the frequency distributions of performance indices for course-keeping and yaw-checking abilities is different from those in Figure 3. The shapes of the frequency distributions are close to the Weibull distribution rather than normal distribution. The same tendency was reported by Woodward [7]. Furthermore, the range of variation of the three indices shown in Figure 3 is different remarkably. The values of 1 st overshoot angle for $10^{\circ} / 10^{\circ}$ zigzag manoeuvre distribute between $35 \%$ and $35 \%$ while the most of 1 st overshoot angle for $20^{\circ} / 20^{\circ}$ exist in the range of $\pm 6 \%$. Therefore, it can be said that zigzag manoeuvre is more sensitive for hydrodynamic coefficients and the mechanism of these phenomenon should be investigated in detail.

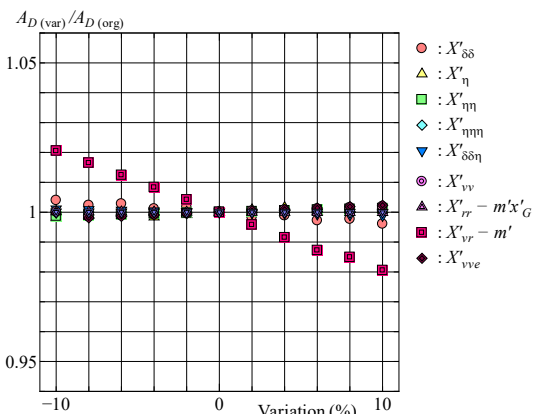

(a) Effects of $X^{\prime}$ derivatives on advance

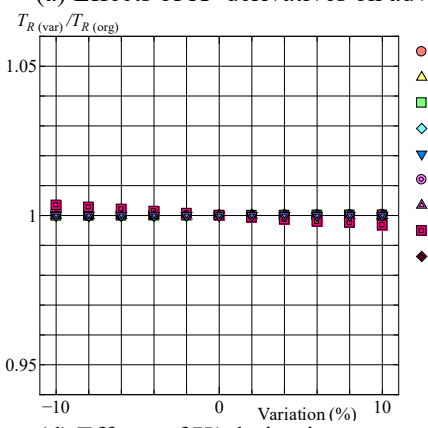

(d) Effects of X' derivatives on transfer

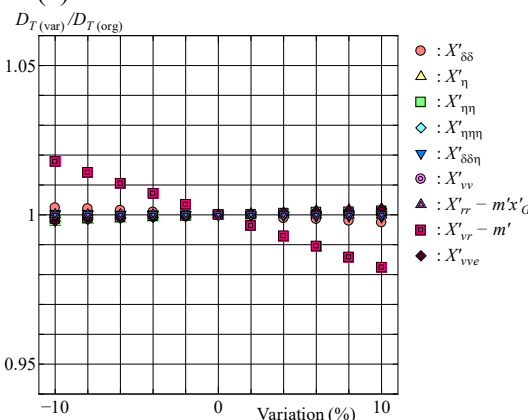

(g) Effects of $X$ ' derivatives on tactical diameter

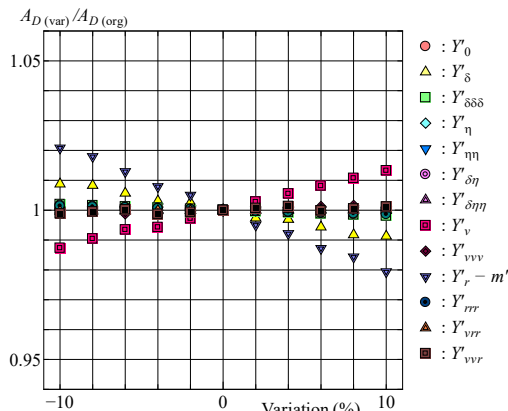

(b) Effects of Y' derivatives on advance

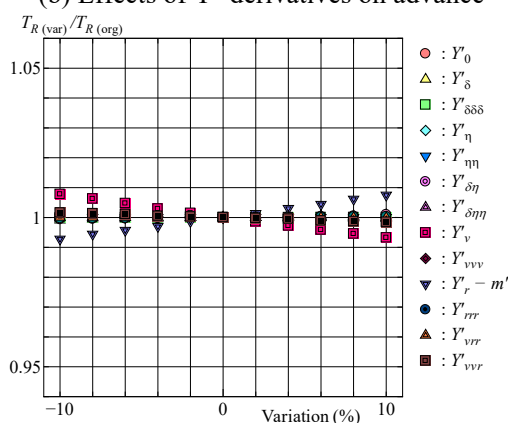

(e) Effects of Y' derivatives on transfer

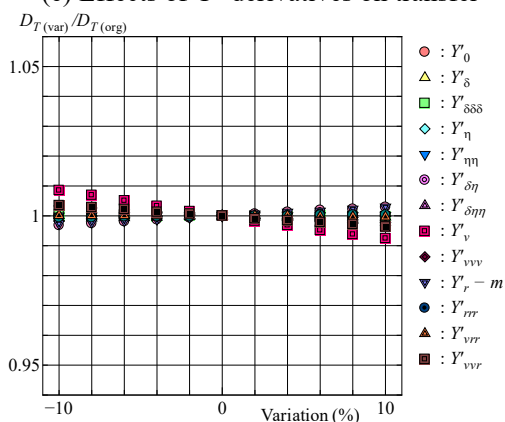

(h) Effects of $Y^{\prime}$ derivatives on tactical diameter

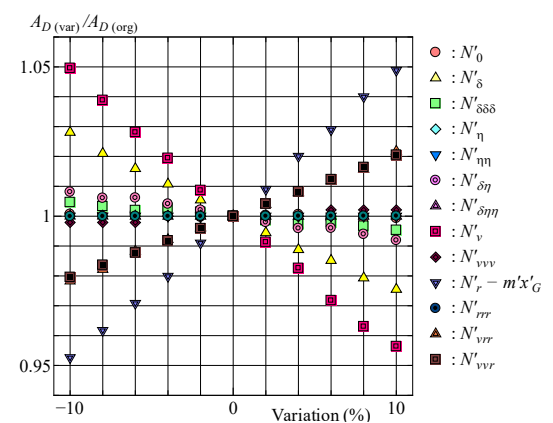

(c) Effects of N' derivatives on advance

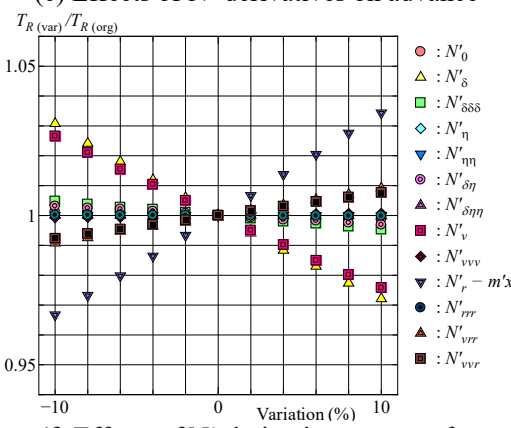

(f) Effects of N' derivatives on transfer

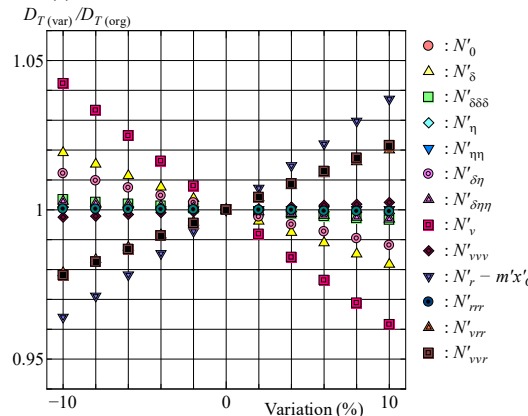

(i) Effects of N' derivatives on tactical diameter

Figure 1: Manoeuvring coefficients for Whole Ship Model. 
As shown in Figs. 1 and 2, there are some hydrodynamic coefficients which have dominant effects on performance indices. According to the observation on Figure 1 shown in the previous section, it was shown that $N_{r}^{\prime}-m^{\prime} x_{G}^{\prime}$ and $N_{v}^{\prime}$ had a dominant effect on the advance, transfer and tactical diameter of a turning circle. Similarly, $Y_{r}^{\prime}-m^{\prime}, Y_{v}^{\prime}, N_{r}^{\prime}-m^{\prime} x_{G}^{\prime}$ and $N_{v}^{\prime}$ much affect on the overshoot angles of zigzag manoeuvre. To confirm the degree of their influence, additional Monte Carlo simulation were conducted.

Figure 5 shows the frequency distributions of advance, transfer and tactical diameter when hydrodynamic derivatives except for $N_{r}^{\prime}-m^{\prime} x_{G}^{\prime}$ and $N_{v}^{\prime}$ are changed based on (4). In other words, the values of $N_{r}^{\prime}-m^{\prime} x_{G}^{\prime}$ and $N_{v}^{\prime}$ are remained constant. It can be observed that the range of the distributions of simulated advance, transfer and tactical diameter becomes narrower comparing with those shown in Figure 3. It proves that $N_{r}^{\prime}-m^{\prime} x_{G}^{\prime}$ and $N_{v}^{\prime}$ have big impacts on the simulated.

The frequency distributions of overshoot angles obtained by the additional Monte Carlo simulations for $10^{\circ} / 10^{\circ}$ and $20^{\circ} / 20^{\circ}$ zigzag manoeuvres are presented in Figure 5. In these simulations, $Y_{r}^{\prime}-m^{\prime}, Y_{v}^{\prime}, N_{r}^{\prime}-m^{\prime} x_{G}^{\prime}$ and $N_{v}^{\prime}$ are remained constant. Even though the values of other hydrodynamic derivatives are changed based on (4), overshoot angles obtained by Monte Carlo simulation converge around each mean value. Therefore, it can be understood that the wide distribution of overshoot angles with the variation of hydrodynamic derivatives are mainly caused by the four linear derivatives $Y_{r}^{\prime}-m^{\prime}, Y_{v}^{\prime}, N_{r}^{\prime}-m^{\prime} x_{G}^{\prime}$ and $N_{v}^{\prime}$ and the influence of the other hydrodynamic derivatives are relatively small.

\section{CONCLUSION}

The investigation of sensitivity analysis using whole ship model had been conducted to understand which hydrodynamic coefficients should be most paid attention when performance indices are obtained by numerical simulations. Monte Carlo Simulation was conducted to prove which coefficients have a big impact on the simulated performance indices. It is found that the variation of $\mathrm{N}^{\prime}$ has the biggest influence for turning ability, course-keeping and yaw-checking abilities. Even though there still exists an influence from variation of $\mathrm{X}^{\prime}$ and $\mathrm{Y}^{\prime}$, the values of performance indices for turning ability are not changed so much. On the other hand, there is no impact at all from the variation of $\mathrm{X}^{\prime}$ for zigzag manoeuvre. This simulation result is corresponding with the Monte Carlo simulation which was carried out to prove which hydrodynamic coefficients have a dominant effect. It can be said that zigzag manoeuvre should be paid more attention because the shapes of the frequency distributions of 1 st and 2 nd overshoot angles are close to the Weibull distribution rather than normal distribution.

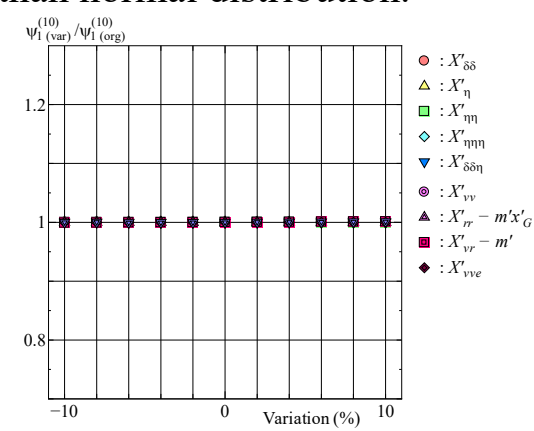

(a) Effects of X' derivatives on 1st ovs of $10^{\circ} / 10^{\circ} \mathrm{z}$

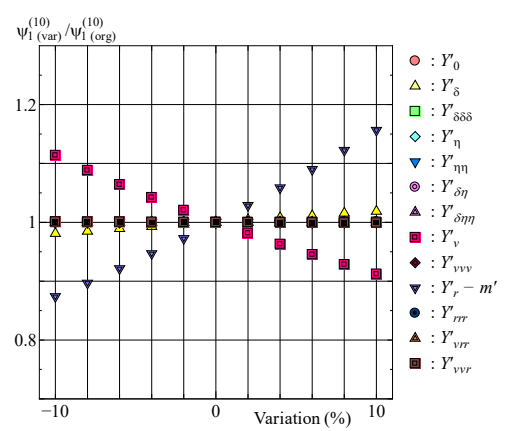

(b) Effects of $Y^{\prime}$ derivatives on $1^{\text {st }}$ ovs of $10^{\circ} / 10^{\circ}$ $\mathrm{z}$

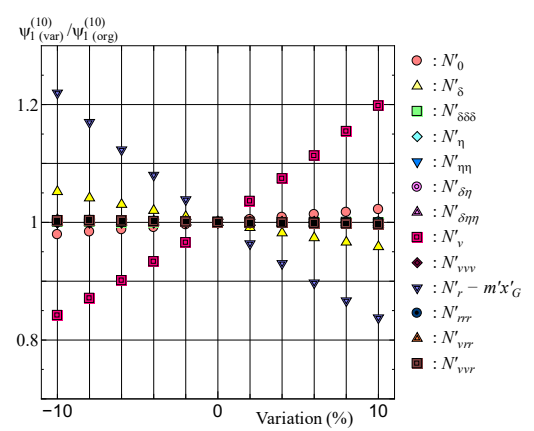

(c) Effects of $N^{\prime}$ derivatives on $1^{\text {st }}$ ovs of $10^{\circ} / 10^{\circ}$ 


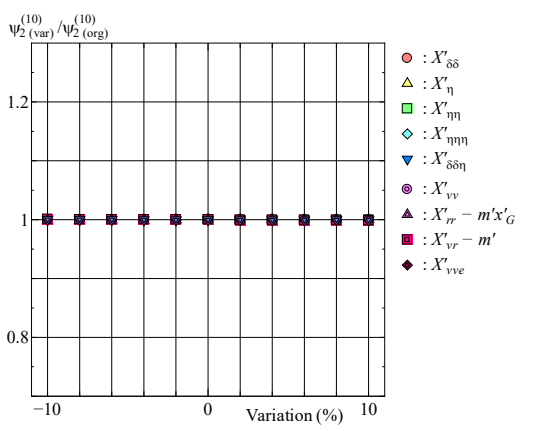

(d) Effects of $X^{\prime}$ derivatives on $2^{\text {nd }}$ ovs of $10^{\circ} / 10^{\circ} \mathrm{z}$

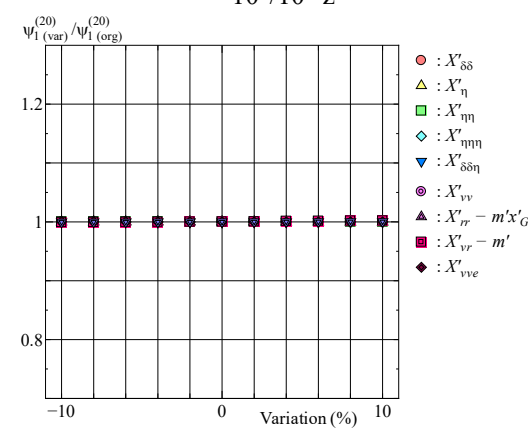

(g) Effects of X' derivatives on 1st ovs of $20^{\circ} / 20^{\circ} \mathrm{z}$

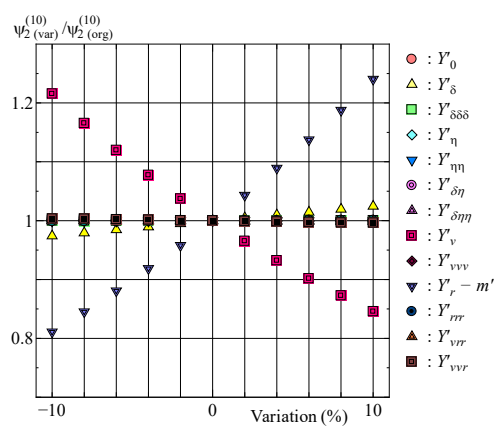

(e) Effects of Y' derivatives on 2nd ovs of $10^{\circ} / 10^{\circ} \mathrm{z}$

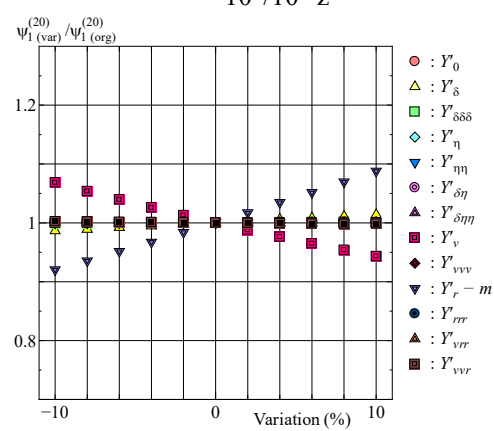

(h) Effects of Y' derivatives on 1st ovs of $20^{\circ} / 20^{\circ} \mathrm{z}$

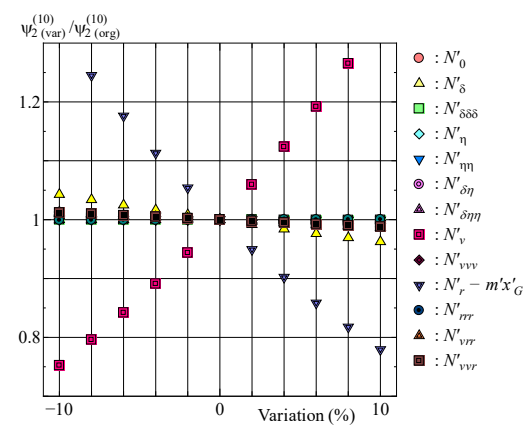

(f) Effects of N' derivatives on 2nd ovs of $10^{\circ} / 10^{\circ} \mathrm{z}$

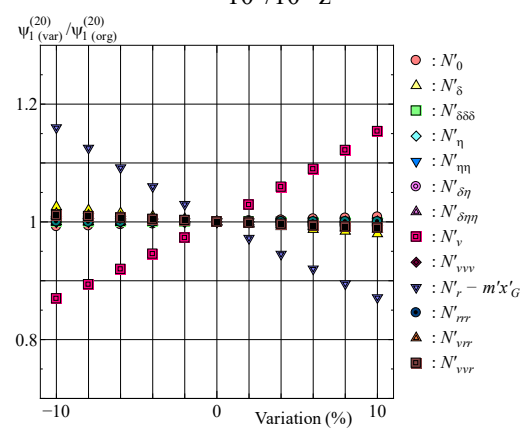

(i) Effects of $\mathrm{N}^{\prime}$ derivatives on 1st ovs of $20^{\circ} / 20^{\circ} \mathrm{z}$

Figure 2: Manoeuvring coefficients for Whole Ship Model.

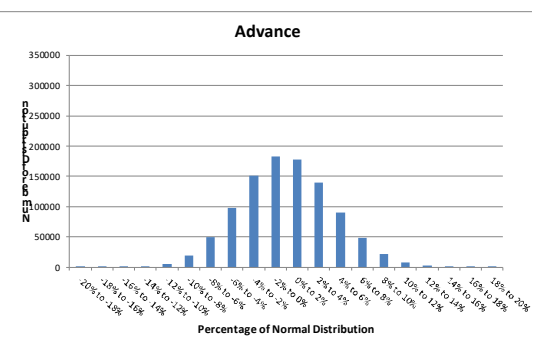

(a) Advance

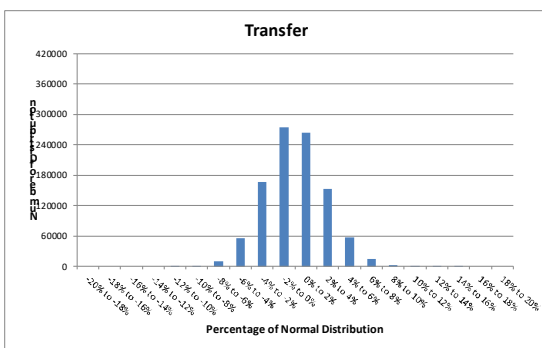

(b) Transfer

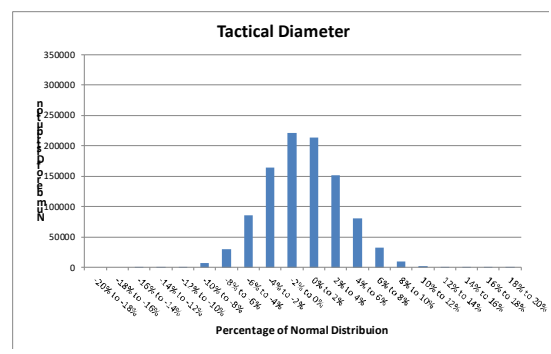

(c) Tactical diameter

Figure 3: Results of Monte Carlo Simulation for turning ability.

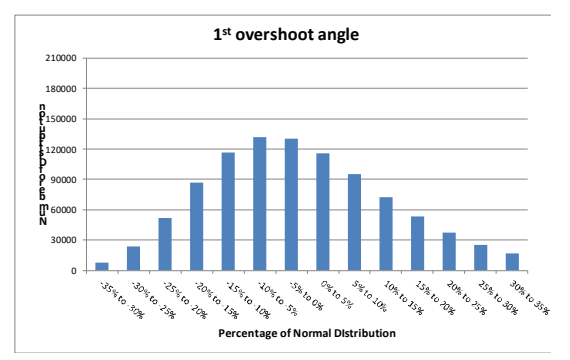

(a) $1^{\text {st }}$ overshoot angle of $10^{\circ} / 10^{\circ}$ zigzag

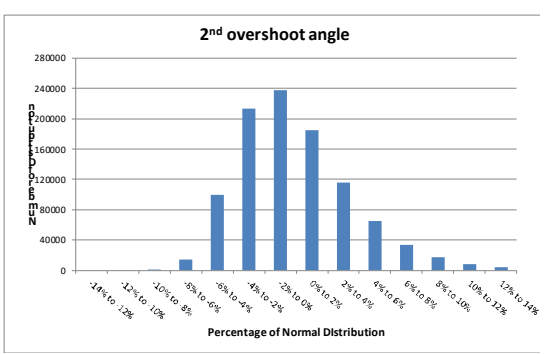

(b) $2^{\text {nd }}$ overshoot angle of $10^{\circ} / 10^{\circ}$ zigzag

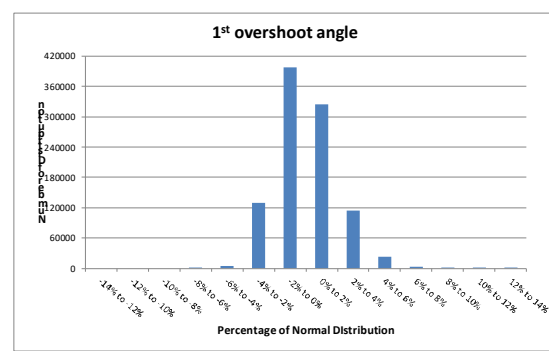

(c) $1^{\text {st }}$ overshoot angle of $20^{\circ} / 20^{\circ}$ zigzag

Figure 4: Result of Monte Carlo Simulation for zigzag manoeuvre 


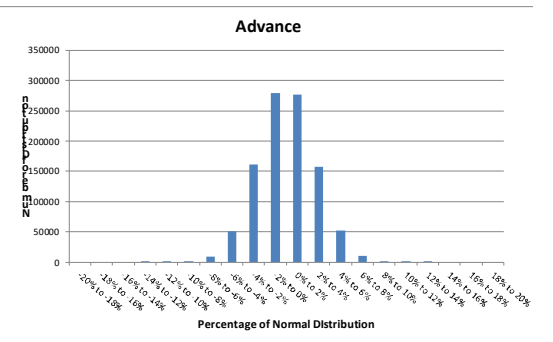

(a) Advance

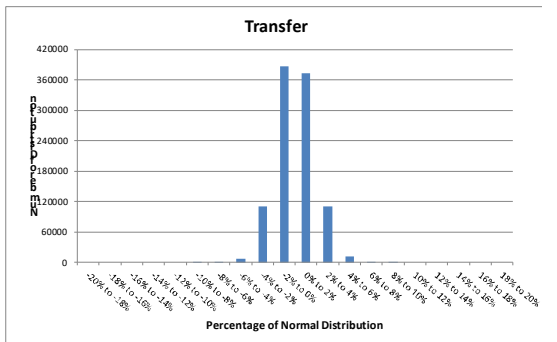

(b) Transfer

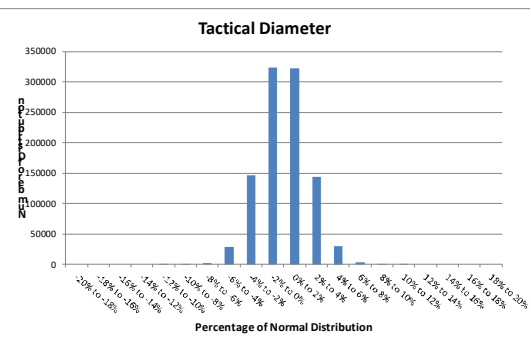

(c) Tactical diameter

Figure 5: Result of additional Monte Carlo Simulation without the variation of $N_{r}^{\prime}-m^{\prime} x_{G}^{\prime}$ and $N_{v}^{\prime}$

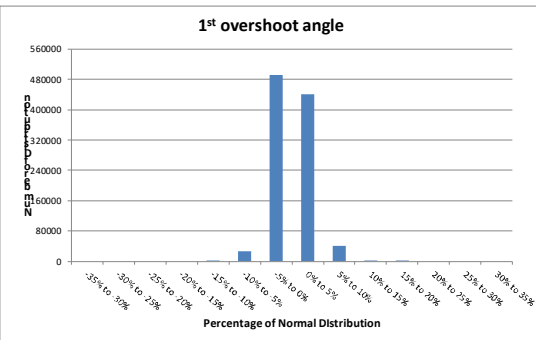

(a) $1^{\text {st }}$ overshoot angle of $10^{\circ} / 10^{\circ}$ zigzag

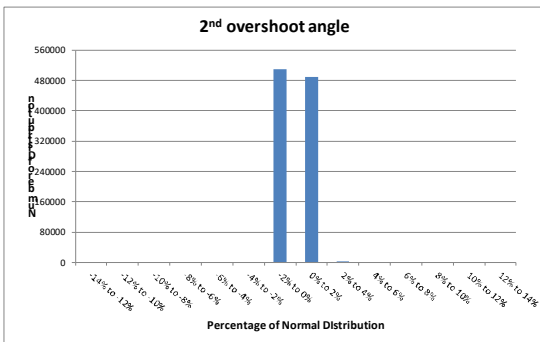

(b) $2^{\text {nd }}$ overshoot angle of $10^{\circ} / 10^{\circ}$ zigzag

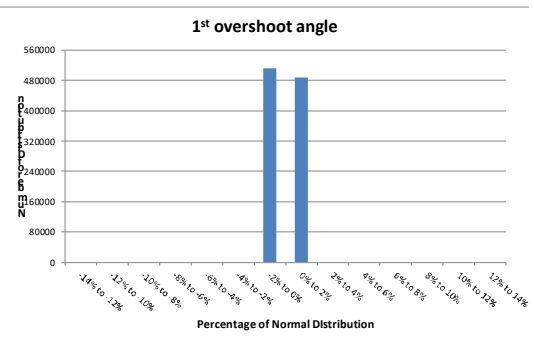

(c) $1^{\text {st }}$ overshoot angle of $20^{\circ} / 20^{\circ}$ zigzag

Figure 6: Result of additional Monte Carlo Simulation without the variation of $Y_{r}^{\prime}-m^{\prime}, Y_{v}^{\prime}, N_{r}^{\prime}-$ $m^{\prime} x_{G}^{\prime}$ and $N_{v}^{\prime}$

\section{ACKNOWLEDGEMENT}

This research is fully supported by Indonesia Endowment Fund for Education (LPDP)

\section{References}

[1] [1] IMO, 2002. Standards for Ship Manoeuvrability, Annex 6 Resolution MSC.137(76) (adopted on 4 December 2002) MSC76/23/Add.1..

[2] ITTC, 2017. Proceeding of 28th ITTC-Volume 1, The Manoeuvring Committee.

[3] Sung, Y. J., Park, S. H., Ahn, K.S.,Chung, S. H., Shin, S. S.and Jun, J. H., 2014, "Eva-luation on Deep Water Manoeuvring Per-formances of KVLCC2 based on PMM Test and RANS Simulation”, SIMMAN 2014 (Preprint), Copenhagen, Denmark, 6 pp.

[4] Shin, S. S., Ahn, K. S., Sung, Y. J. and Oh, S. H., 2014, "A Study on Effect of the Self-Propulsion Points in PMM Tests for KVLCC's Manoeuvrability", MARSIM 2014.

[5] ITTC - Recommended Procedure and Guidelines: Uncertainty Analysis for Manoeuvring Predictions based on Captive Manoeuvring Tests, 7.5-02-06-04, p.33, Effective date 2017, Rev. 2.

[6] Son, K. and Nomoto, K., 1981, “On the Coupled Motion of Steering and Rolling of a High Speed Container Ship”, Journal of the Society of Naval Architects of Japan, Vol. 1981, No,150, pp.232-244

[7] Woodward, M. D., 2014, "Evaluation of Interfacility Uncertainty for Ship Manoeuvring Performance Prediction”, Ocean Engineering, Vol. 88, pp. 598-606.

[8] Wang, X.G., Zou, Z.-J., Xu, F. and Ren, R.-Y., 2014, "Sensitivity Analysis and Para-metric Identification for Ship Manoeu-vring in 4 Degrees of Freedom", Journal of Marine Science and Technology, Vol. 19, pp. 394-405.

[9] Dash, A. K., Nagarajan, V. and Sha, O. P., 2015, "Uncertainty Assessment for Ship Maneuvering Mathematical Model”, International Shipcbuilding Progress, Vol. 61, pp. 129-161. 